LA-UR- 95-3106



Submitted to:

IEEE/Pulsed Power

Albuquerque, NM

July 1995



Los Alamos National Laboratory, an affirmative action/equal opportunity employer, is operated by the University of California tor the U.S. Department of Energy under contract W-7405-ENG-36. By acceptance of this article, the publisher recognizes that the U.S. Govemment retains a nonexclusive, royalty-free license to publish or reproduce the published form of this contribution, or to allow others to do so, for U.S. Govemment purposes. The Los Alamos National Laboratory requests that the publisher identify this article as work performed under the auspices of the U.S. Department of Energy. 


\section{DISCLAIMER}

Portions of this document may be illegible in electronic image products. Images are produced from the best available original document. 


\title{
Comparing Calculated and Measured X-Ray Images
}

\author{
W. Matuska, J. Benage, G. Idzorek, C. Lebeda, D. Peterson \\ Los Alamos National Laboratory, Los Alamos, NM, 87544
}

\begin{abstract}
In recent years 2-dimensional radiation-magneto-hydrodynamic (RMHD) calculations have done quite well in matching some important observed parameters of a z-pinch implosion. As we gain experience, we field more complex experiments to compare with calculations. Here we discuss both time dependent and time integrated $x$-ray imaging on Pegasus. Images, using similar filters, are calculated and compared with the data. We also apply some image enhancement to the data.
\end{abstract}

\section{Introduction}

Much progress has been made in recent years in matching 2-dimensional (2-D) radiation-magneto-hydrodynamic (RMHD) calculations to data obtained from z-pinch implosions on Pegasus and Procyon, Los Alamos facilities. Pegasus 2-D RMHD calculations (1) often quantitatively match the observed current wave form very accurately. Calculated instability patterns, spikes and bubbles, resemble time-dependent, visible observed images, but no quantitative comparison has been made. The calculated total radial energy out often can compare well with the energy and radiated power measured with a bolometer. The calculated, time-dependent radial power out often compares well with the shape of the low photon energy (where most of the radiated energy is found) $\mathrm{x}$-ray diode (XRD) signals. Peterson (1) normalizes the calculated radiative power curves for the XRD comparisons. Calculated time dependent $\mathrm{X}$-ray spectra ${ }^{(2)}$ have been found to be in qualitative agreement with spectra from a transmission grating spectrometer (TGS). XRD signals, inferred from these calculated spectra, have been put on an absolute scale with XRD data and the comparison was fair. Not only do we compare calculations with experiment, we compare experiment with experiment since Pegasus has 18 equally-spaced ports and we are able to field many diagnostics. This has lead to finding unexpected inconsistencies, hence problems, with diagnostics. Here we address $x$-ray imaging diagnostics. Progress has been made in some areas, especially stripline camera blooming, since the data considered here were obtained, but this is the most recent complete set of data and calculations available.

\section{Calculations}

The 2-D RMHD calculation considered here is the best match to Pegasus experiment PegII-25 ${ }^{(1)}$. The calculation is of a cylindrically symmetric geometry with rectangular Eulerian zones. Spectra are calculated along rays through the center of each of the zones, using the transport equation ${ }^{(2)}$, as illustrated in Fig. 1. Such a set of spectra is calculated at a specified time. Contributions due to scattering are estimated to be very low and are not included in these calculations. The calculated image is formed by folding each calculated spectrum with the response of the imaging system and then plotting the resulting intensities on a 2-D grid. This image is expanded by reflecting about $\mathrm{r}=0$ for ease in comparing with data. Time integrated images are calculated by simply doing a time interval weighted sum of images calculated at many different times. The radial power radiated along a line-of-sight, as a function of time, is also calculated. This is done by summing all calculated spectra, multiplied by zone area, and then integrating over photon energy. This power curve, shown in Fig. 2, puts image times into perspective.

\section{Stripline and Pinhole Cameras}

The stripline camera, shown schematically in Fig. 3, records x-ray images through four different filters at five different times. The times of these images are indicated in Fig. 2 . The filters are 0.65 micron $\left(175.5 \mu \mathrm{g} / \mathrm{cm}^{2}\right) \mathrm{Al}, 2.0$ micron parylene-N, 1.27 micron $\mathrm{Ni}$, and 7.0 micron Al. The first two filters allow transmission of soft $\mathrm{x}$-rays and hence image most, if not all, of the pinch. The last two filters image only the hottest part of the pinch since they only transmit the harder $\mathrm{x}$-rays. Here we will concentrate on the $175.5 \mu \mathrm{g} / \mathrm{cm}^{2}$ image at the fourth time. 


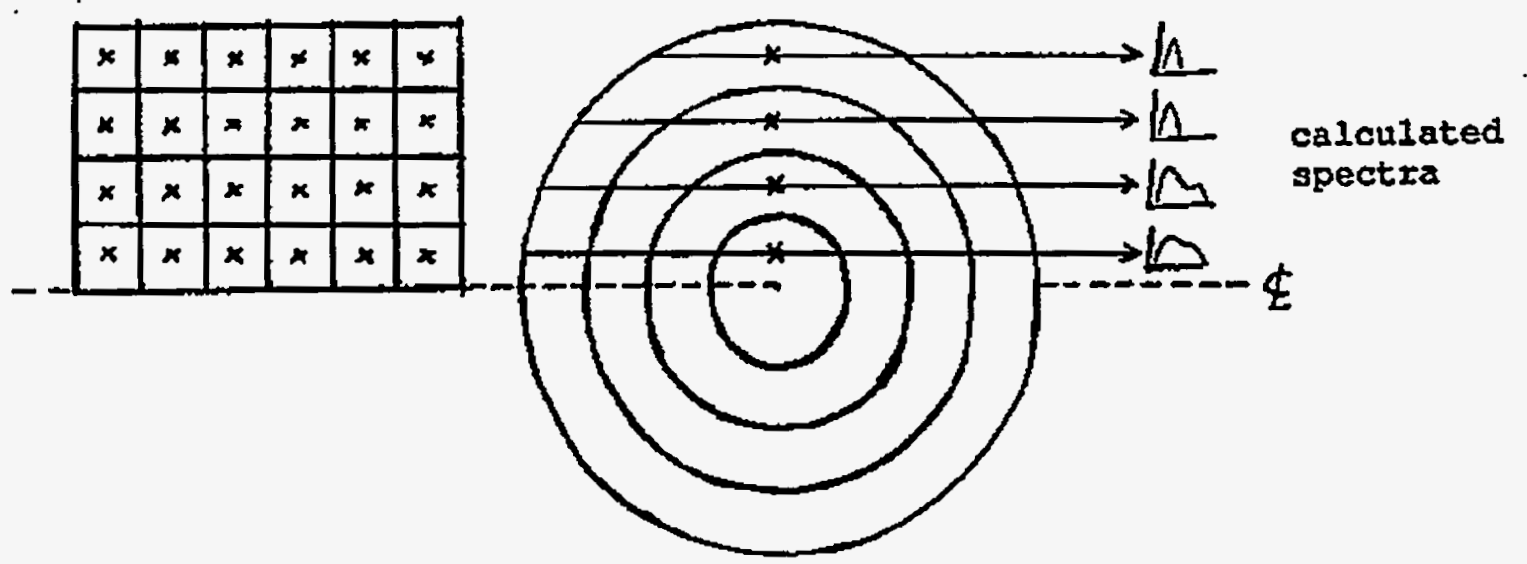

Fold each of the spectra with resposse

function to obtain calculated pixel intensities.

calculated jmage



(reflect here)

Figure 1. Schematic illustrating method used to calculate images.

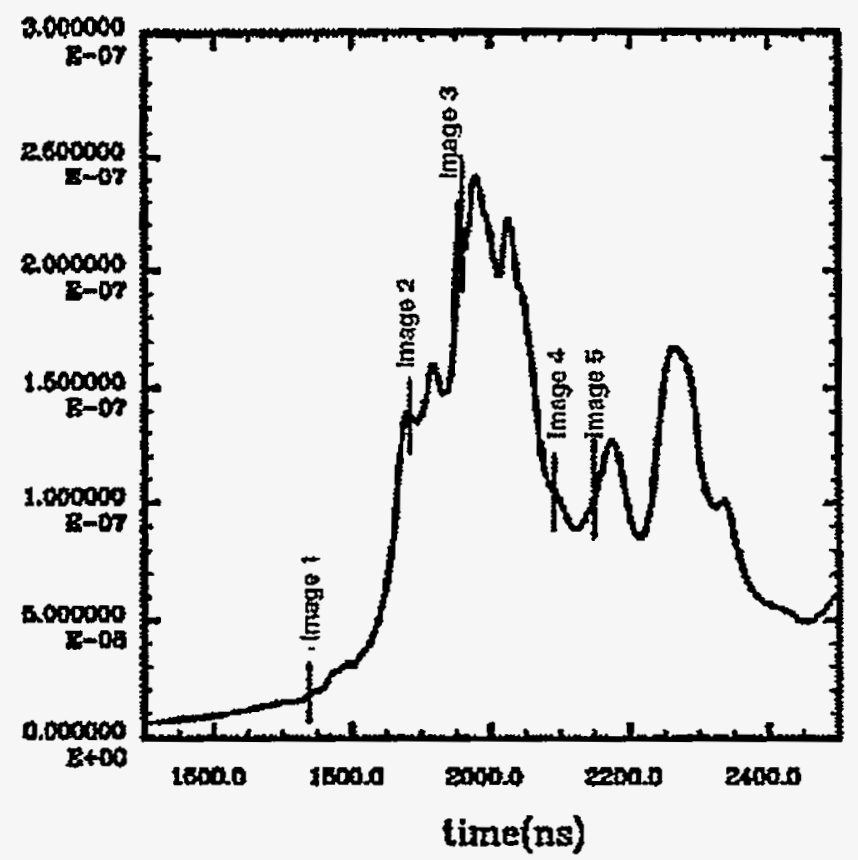

Figure 2. Locations of stripline camera images on a calculated power curve. 
Figure 3. Schematic illustrating stripline camera layout.

$X$-rays, after they passed through the pinhole and $\mathrm{X}$-ray filter, strike the gold front surface of the microchannel plate where they knock off electrons. When the pulsed voltage is on (about $5 \mathrm{~ns}$, which defines the exposure time), the electrons follow the electric field lines generated by this voltage and are thus directed into the microchannels, where each electron avalanches to create $10^{3}$ to $10^{4}$ electrons. These electrons exit the opposite side of the microchannel plate and traverse a gap, across which is a 3 to $4 \mathrm{KV}$ bias voltage, before striking a phosphor screen. Here they create visible light which is recorded on film.

The exact cause of stripline camera blooming is not known, but we found on Pegasus experiments ${ }^{(3)}$ subsequent to the one discussed here and on Saturn, a Sandia National Laboratory z-pinch machine, that decreasing the pulsed voltage greatly reduces, and perhaps even eliminates, the blooming. The degree of blooming is sensitive to the pulsed voltage, but there is the danger of getting no image at all if the voltage is too low. The blooming is also a function of the source intensity. These observations lead us to believe that as the pulsed voltage is increased, the electric field lines extend farther along the microchannel plate, causing electrons to enter channels substantial distances from where they were created, and thus being the source of blooming.

We do not know exactly the extent of the blooming, but we can get an idea from the 1-D slice of intensities (line out) near the middle of the stripline corresponding to the fourth time, shown in Fig.4.

One can tell by inspecting the data that the images are greatly distorted by blooming. This is demonstrated in Fig. 4 . Ideally, intensities should be zero everywhere except for the four images, which can never be wider than the window. (Windows are $3 \mathrm{~cm}$ by $2 \mathrm{~cm}$ rectangular apertures in the current return path to allow diagnosing the pinch.) Here maximum intensities outside the window is about a third maximum image intensity. The blooming appears not to be alike in both directions. We will use the intensity fall-off to the right of the 0.65 micron $\mathrm{Al}$ image in the data analysis below. 
The time integrated pinhole camera is a standard pinhole camera which makes seven images, one through each of seven $0.1 \mathrm{~mm}$ diameter pinholes. The pinholes, and images, are arranged six in a circle with one in the center. A different $\mathrm{x}$-ray filter is associated with each pinhole. The $\mathrm{x}$-rays shine onto a BC-422 scintillator which is in contact with TMAX-400 film. The scintillator has a $10 \mu \mathrm{g} / \mathrm{cm}^{2} \mathrm{Al}$ overcoat.

For this comparison between stripline and pinhole cameras, we selected the images whose responses were the most similar. The responses for the two cameras can never be identical, but they can be more alike than those used, which are shown in Fig. 5. The microchannel plate and phosphor screen construction of the stripline camera causes the response to decrease with increasing photon energy, whereas the pinhole camera scintillator converts $x$-rays into equal power of visible light, hence the pinhole camera response is simply proportional to the $\mathrm{x}$-ray transmission through the filter. For an Al filter as shown in Fig. 5, the first lobe of the pinhole camera response can never have greater amplitude than the second lobe, but for a thinner filter, the relative amplitudes of the two lobes will be more alike. The preferred filter is $175.5 \mu \mathrm{g} / \mathrm{cm}^{2} \mathrm{Al}$ (same as the stripline camera), but care must be taken since this filter is prone to tiny holes.

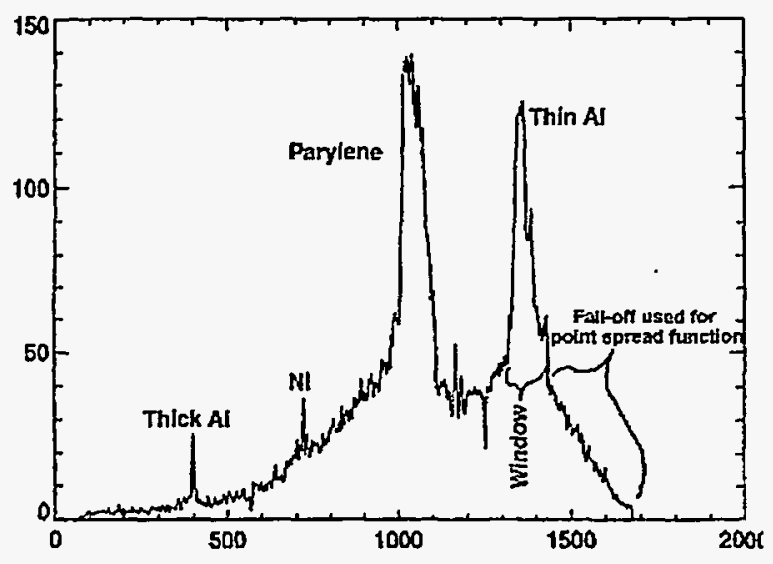

Figure 4. A 1-D slice of intensities near the middle of the stripline corresponding to the fourth time. The images for the four images are marked as is the region used for the point spread function.

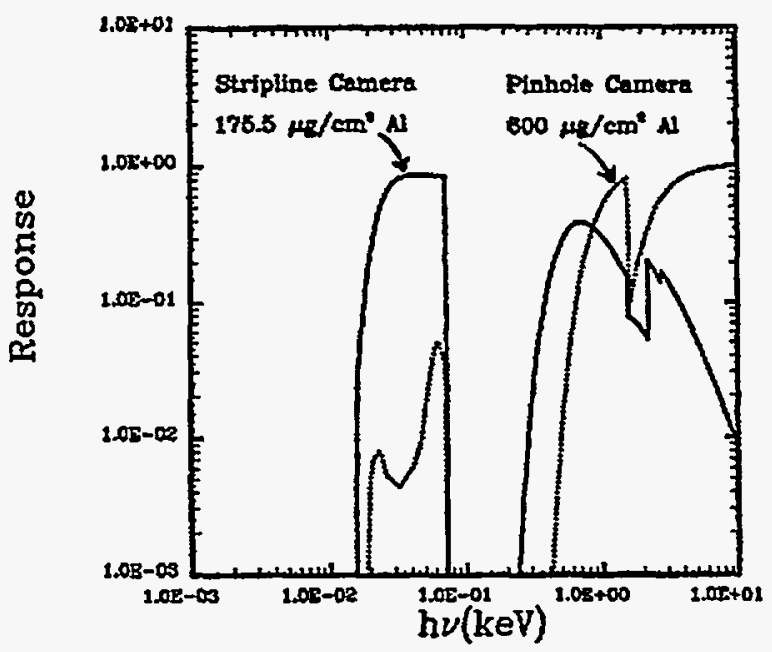

Figure 5. Aluminum filter response functions for the stripline and pinhole cameras.

\section{Comparison of Images}

All experimental images and calculated images are in relative intensities, not film density. No attempt is made to put these images on an absolute scale, and all images are normalized to their maximum value. All images here are treated in a digitized form. The stripline camera image ( 0.65 micron Al filter) at the fourth time is selected for this analysis, even though the third time is nearer peak power, since the fourth time image is the only image near peak power which clearly shows a pinch. This image, shown in Fig. $6 \mathrm{a}$, also reveals the blooming, which was so obvious in Fig. 4, as well as isolated spots of high frequency noise. This noise was eliminated with a $5 \times 5$ median filter, and the resulting image is shown in Fig. $6 \mathrm{~b}$. A broad pinch is seen, believed to be much wider than the actual pinch. Also, if there were no blooming, all intensities outside the window area should be zero. Techniques exist to remove a point spread function from a blurred image and thus reconstructing an image more representative of the source; however, since the extent of the blooming is so great here and the true shape of the point spread function is unknown, we do not attempt such a reconstruction.

An image corresponding to the fourth time is calculated by the method described above and shown in Fig. 6c. Here the pinch appears much narrower than in Fig. $6 \mathrm{~b}$, and the intensities away from the pinch are very small. We use a portion of the line out in Fig. 4 to estimate a point spread function to approximate the blooming. Figure $6 \mathrm{~d}$ is obtained by applying this point spread function to Fig. $6 c$. By comparing Figs. $6 \mathrm{~b}$ and $6 \mathrm{~d}$, which is the best that presently can be done, we see that data and calculation resemble each other. 

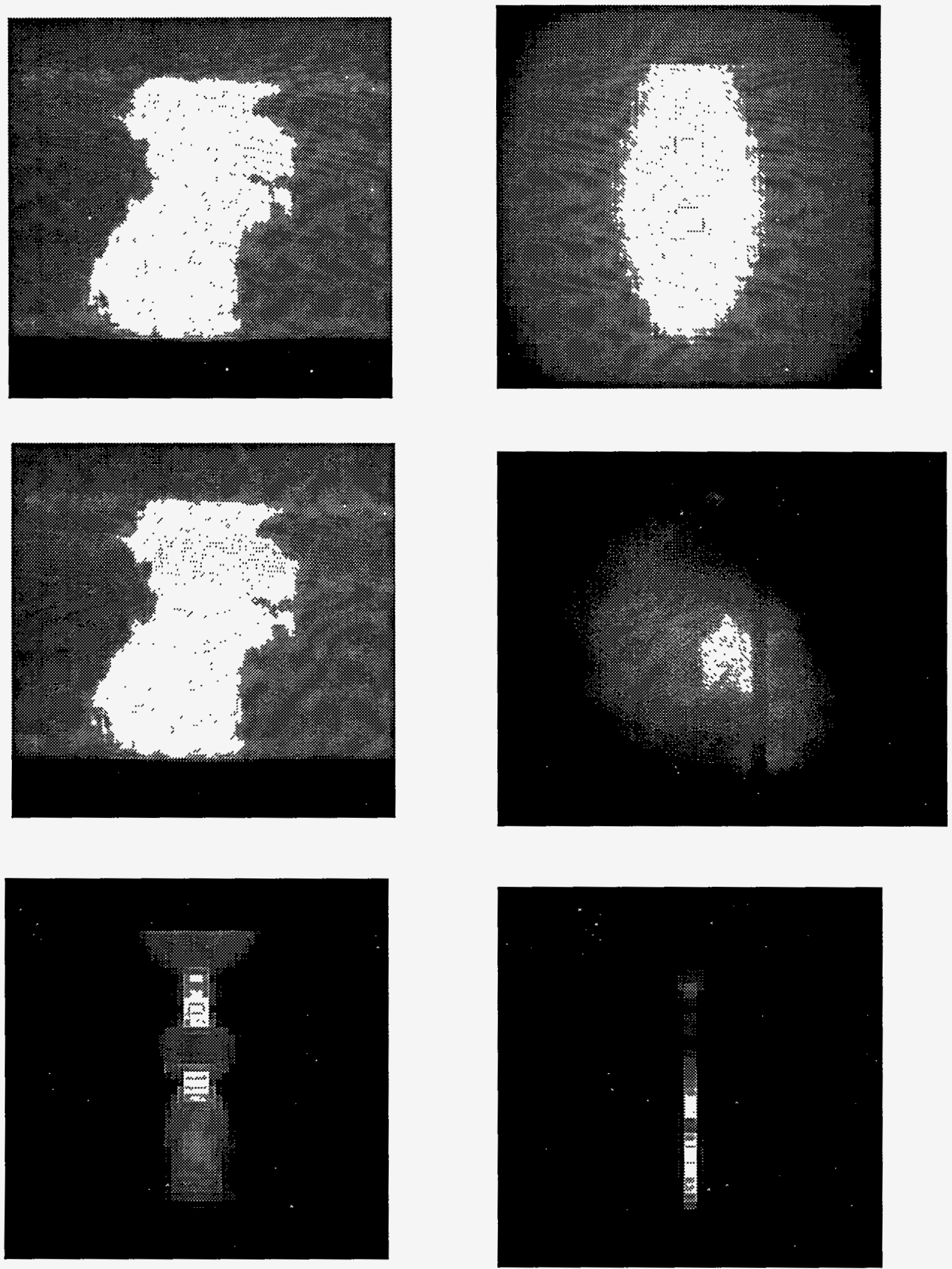

Figure 6. All images are $3.0 \mathrm{~cm}$ by $2.5 \mathrm{~cm}$. a) Raw stripline camera image, b) $5 \times 5$ median filter applied to raw image, c) calculated stripline camera image, d) point spread function applied to calculated image, e) pinhole camera image, and f) calculated pinhole camera image. 
The pinhole camera images are a simple comparison between data and calculations. The experimental time integrated image is shown in Fig. 6e. The vertical line of low intensity to the right of the high intensity area is a wire in the window to locate the axis of the foil. Due to parallax, the pinch appears off axis. To obtain the calculated image in Fig. 6f, images were calculated, using the method described above, at approximately $5 \mathrm{~ns}$ intervals and then integrated over time.

The calculated single time image in Fig. $6 \mathrm{c}$ and the and the calculated time integrated image in Fig. $6 \mathrm{f}$ both show the pinch to be concentrated along the axis. The pinch in the stripline camera image, Fig. $6 \mathrm{~b}$, also appears to be concentrated along the axis. The calculated single time image is larger in radius, since it is after the peak, than the time integrated image which gets most of its contribution from the peak. The radius of the pinch actually seen by the stripline camera is of course very uncertain. The pinhole camera image is a bit of a puzzle. The pinhole camera image indicates an oval pinch which is larger in the radial direction than along the pinch axis, which is qualitatively different from calculations and the stripline camera image. The reason for this difference is unknown, but since the responses for the two cameras are somewhat different and the actual spectrum at each pixel is not known (we relied on calculations), this difference in image shape could be real. On the other hand, cross checking diagnostics is a good way to uncover unanticipated problems.

\section{Conclusions}

Much progress has been made in recent years to match 2-D RMHD calculations to data obtained from z-pinch implosions. Equally as important, the 18 equally spaced radial ports on Pegasus allow us to cross check diagnostics, which is very useful in evaluating the accuracy of the data. Here we found that calculations and a single time stripline camera image show similarities, while the time integrated pinhole camera image appears to be different. We emphasize that this three way cross check is at a very preliminary stage. If permitted by our pulsed power program policy, we will work to understand these differences and correct any problems we may find with calculations or diagnostics.

\section{References}

1. D.L. Peterson, R.L. Bowers, C.F. Lebeda, W. Matuska, J. Benage, G. Idzorek, H. Oona and J. Stokes, "Comparison and Analysis of 2-D Simulation Results with Two Implosion Radiation Experiments on the Los Alamos Pegasus I and Pegasus II Capacitor Banks," these proceedings.

2. W. Matuska, H. Lee, R. Hockaday and D. Peterson, "Source to Detector Spectrum Transformation and its Inverse for the Pegasus Z-Pinch,” AIP Conference Proceedings 299, Dense Z-Pinches, Third International Conference, London, United Kingdom 1993, 525.

3. H. Oona, D.L. Peterson, W. Anderson, J. Benage, G. Idzorek, M.L. Yapuncich, C. Findley, J. Shlachter, J.H. Goforth, J.C. Cochrane and B. Anderson, "Instabilities in Foil Implosions and the Effect on Radiation Output," these proceedings.

\section{DISCLAIMER}

This report was prepared as an account of work sponsored by an agency of the United States Government. Neither the United States Government nor any agency thereof, nor any of their employees, makes any warranty, express or implied, or assumes any legal liability or responsibility for the accuracy, completeness, or usefulness of any information, apparatus, product, or process disclosed, or represents that its use would not infringe privately owned rights. Reference herein to any specific commercial product, process, or service by trade name, trademark, manufacturer, or otherwise does not necessarily constitute or imply its endorsement, recommendation, or favoring by the United States Government or any agency thereof. The views and opinions of authors expressed herein do not necessarily state or reflect those of the United States Government or any agency thereof. 